\title{
Quality of life during formoterol treatment: comparison between asthma-specific and generic questionnaires
}

\author{
T. van der Molen*, M.R. Sears+, C.S. de Graaff $;$, D.S. Postma*, B. Meyboom-de Jong* \\ for the Canadian and the Dutch Formoterol Investigators
}

\begin{abstract}
Quality of life during formoterol treatment: comparison between asthma-specific and generic questionnaires. T. van der Molen, M.R. Sears, C.S. de Graaff, D.S. Postma, B. Meyboom-de Jong for the Canadian and the Dutch Formoterol Investigators. OERS Journals Ltd 1998.

ABSTRACT: This study aimed to investigate the effect of treating patients with moderate asthma with formoterol on quality of life $(\mathrm{QoL})$ and to compare several questionnaires in their ability to detect changes in QoL.

In an eight month multicentre, randomized, placebo-controlled trial, patients with

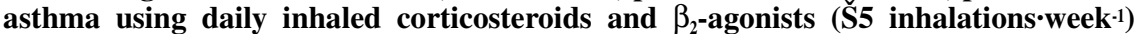
were randomized to 6 months treatment with formoterol $24 \mu \mathrm{g}$ b.i.d. (by Turbuhaler ${ }^{\circledR)}$ or a matching placebo. Patients recorded symptoms (maximal score 21) and peak expiratory flow (PEF) twice daily. QoL was measured with two asthma-specific questionnaires, the Asthma Quality of Life Questionnaire (AQLQ) and the Living with Asthma Questionnaire (LWAQ), and with two generic QoL forms, i.e. the Short Form 36 (SF36) and the Psychological and General Well-Being scale (PGWB) at randomization and after 6 months of treatment.

Out of 110 patients studied, 56 received formoterol and 54 placebo. Mean baseline forced expiratory volume in one second was $65 \%$ predicted. Mean morning PEF was 369 L·min'-1. Mean total symptom score was 3.6. Morning PEF increased $(26.8 \mathrm{~L} \cdot$ $\left.\min ^{-1}, p=0.0001\right)$ and symptoms decreased $(-1.23, p=0.012)$ in the formoterol group in contrast to placebo. QoL improvements were measured in the LWAQ total score (baseline 0.61 , change $-0.05, p=0.048$ ) and the physical construct of the LWAQ (baseline 0.71, change 0.07, $\mathrm{p}=\mathbf{0 . 0 4 4}$ ). The AQLQ and the generic QoL instruments showed no significant changes.

In conclusion, the improvement in quality of life reported after 6 months was very small and only reflected by the Living with Asthma Questionnaire.

Eur Respir J 1998; 12: 30-34.
\end{abstract}

*Groningen University, Groningen, The Netherlands. 'McMaster University, Hamilton, Ontario, Canada. $¥$ Medical Centre Alkmaar, Alkmaar, The Netherlands.

Correspondence: T. van der Molen

Antonius Deusinglaan 4

9713 AW Groningen

The Netherlands

Fax: 31503632964

Keywords: Asthma

$\beta$-agonists

formoterol

quality of life

Received: February 191997

Accepted after revision March 171998

Supported financially by Astra Draco AB Sweden
Measurement of quality of life (QoL) is increasingly being used in clinical intervention studies and has been shown to be a valid indicator in assessing whether a medical treatment is beneficial [1-4]. The assessment of QoL is now regarded as being one of the main outcome variables in clinical studies [5, 6], especially in chronic diseases such as asthma, in which a cure by medical intervention is seldom achieved. In this respect, QoL is referred to as the daily functioning and the subjective wellbeing of the pat-ient. Although asthma-specific QoL questionnaires have been developed only recently, the number of trials in which QoL measurement has been applied is growing rapidly.

Clinical studies to investigate the effectiveness of longacting $\beta_{2}$-agonists have been the first to assess the benefit of QoL, together with objective measures of severity of the disease. Virtually all studies have shown significant improvements in lung function, use of rescue medication to control symptoms, and diary-obtained symptom scores [7-11]. In contrast, improvement in QoL by long-acting $\beta_{2}$-agonist treatment in asthmatic patients is not as clear as improvement in the other measures of disease [2, 12]. Spirometric parameters, symptom scores and use of medication improved as expected, but only one of the wide variety of QoL questionnaires revealed a substantial improvement. Improvements in lung function and symptoms do not always reflect a clear improvement in the recorded QoL. The question is whether this is dependent on the kind of questionnaire used or whether patients do not perceive a substantial improvement in their QoL as recorded on any kind of questionnaire.

In this study, a comparison was made of the performance of two asthma-specific QoL questionnaires and three generic questionnaires with a structured symptom score diary and lung function measurements in order to assess their ability to detect clinical improvement. The aim of the study was to assess which QoL questionnaire was most sensitive to change. The study was part of a multicentre international study on the efficacy of inhaled formoterol in 
mild-to-moderate asthmatic patients using inhaled corticosteroids.

\section{Patients and methods}

\section{Patients}

During a study regarding the efficacy of the long-acting $\beta_{2}$-agonist formoterol (Formoterol Turbuhaler®, Astra Draco, Lund, Sweden), 230 adult asthmatic patients were followed for 8 months. Details about methodology and QoL assessment have been described previously [13, 14]. The present study pertains to the 110 Dutch patients. Patients participated in five centres in The Netherlands, 65 patients recruited from 44 general practitioners and 32 from four outpatient hospital clinics. All asthmatic patients were in a stable phase of the disease and used inhaled corticosteroids regularly and short-acting $\beta_{2}$-agonists as needed, with a minimum of five inhalations in the week before randomization. Patients using theophyllines and oral steroids were excluded. On entering the study, the forced expiratory volume in one second (FEV1) was $>40 \%$ predicted and reversibility after $500 \mu \mathrm{g}$ of terbutaline $>15 \%$ of baseline. The study protocol was approved by the Medical Ethics Committees of all participating centres and patients gave written informed consent.

\section{Study design}

A double-blind placebo-controlled parallel design was used, with a run-in period (4 weeks) to obtain baseline values, a treatment period ( 24 weeks) and a wash-out period (4 weeks). The dose of inhaled corticosteroids taken on entry was maintained at a constant level throughout the study. Patients were treated with formoterol $24 \mu \mathrm{g}$ twice daily or placebo. Terbutaline sulphate $(250 \mu \mathrm{g}$ inhalations via the Turbuhaler $($ ) was allowed as needed for the relief of symptoms. No long-acting $\beta_{2}$-agonists were allowed, with the exception of the study medication. Patients filled in daily diary cards to record daytime symptoms of chest discomfort, sputum production, cough, activity and wheezing on a scale ranging from 0 (none) to 3 (very severe). Night-time symptoms of wheeze and cough were recorded on the same scale, adding up to a total asthma symptom score of 21. The highest of three measurements of peak expiratory flow (PEF) by a Mini-Wright peak flow meter was recorded each morning and evening, together with the use of daytime and night-time bronchodilator and other medication. PEF was measured before inhalation of the study drug and the patients were asked to refrain from the use of terbutaline at least $6 \mathrm{~h}$ before measurements.

\section{Quality-of-life measurements}

QoL was assessed at the end of the run-in period before the start of treatment and at the end of the 6 month treatment period. Two generic QoL instruments were used: the Short Form 36 (SF36) [15, 16] and the Psychological and General Well-Being index (PGWB) [17, 18]. Two asthmaspecific QoL questionnaires were also used: the Living with Asthma Questionnaire (LWAQ) $[19,20]$ and the Asthma Quality of Life Questionnaire (AQLQ) [21, 22]. The AQLQ can be used in a self-administered and an interview-administered form. In this study the interviewadministered form was used. Interviewers were trained by an instructional video tape. The QoL measurements were all performed in the same order after lung-function measurement; first, the AQLQ interview, followed by a booklet with the three other questionnaires. Patients were not allowed to see their previous answers scored during the first visit.

\section{Spirometry}

FEV1, and forced vital capacity (FVC) were measured using calibrated spirometers in the morning on the same day as the four QoL questionnaires were filled in. Reversibility of FEV 1 was tested at entry in the morning between 08:00 and 12:00 h after 500 $\mu \mathrm{g}$ of terbutaline (Bricanyl Turbuhaler®). $\beta_{2}$-agonists were stopped at least $8 \mathrm{~h}$ before the test. Measurements were made in triplicate and the best recorded.

\section{Data analysis}

QoL questionnaires were scored following their respective instructions. The LWAQ was calculated using its two construct subscales [2]. Symptom scores are presented as the mean total symptom scores during 1 day. All diary variables were calculated separately as a mean of the last 14 days before treatment and the last 14 days of the treatment. Morning and evening PEF were the mean of PEF values of morning and evening diary records, respectively. Changes in symptom scores were calculated by taking the absolute difference between mean scores of the last 14 days before treatment and the last 14 days during treatment.

Statistical tests were carried out on an intention-to-treat basis for valid data. Valid questionnaire domains required an answer to at least $80 \%$ of the items, except in the AQLQ "activity" domain, in which the rules of the author were followed [22]. The results of the QoL instruments and the symptom scores were tested for internal consistency, using Cronbach's alpha. All analyses were performed using the Statistical Package for Social Sciences (SPSS Inc., Chicago, IL, USA).

The changes from randomization to the end of treatment period were analysed. Student's t-tests were used for all indices and domains of the QoL questionnaires in order to compare the changes during treatment between the two treatment groups. In addition, Cohen's effect size was calculated for the asthma-specific questionnaires in order to compare questionnaires with different scales.

\section{Results}

\section{Patients}

Patient characteristics and their baseline QoL values are shown in table 1 . Three patients in the formoterol group 
Table 1. - Patient characteristics and baseline values by treatment group

\begin{tabular}{lcc}
\hline & Formoterol & Placebo \\
\hline Patients n & 56 & 54 \\
Age yrs & $40.8 \pm 13.2$ & $46.4 \pm 14.2$ \\
Male n & 28 & 28 \\
Atopy \% & 68.8 & 66.6 \\
FEV1 \% pred & $65.9 \pm 17.2$ & $64.7 \pm 16.9$ \\
Reversibility in FEV1\% baseline & $25.2 \pm 11.8$ & $26.3 \pm 13.1$ \\
PEF morning L·min ${ }^{-1}$ & $376 \pm 108.9$ & $361 \pm 95.1$ \\
Symptom score $(0-21)$ & $3.76 \pm 2.9$ & $3.5 \pm 2.8$ \\
$\beta_{2}$-agonist use inhalations·day-1 & $1.86 \pm 1.4$ & $1.68 \pm 0.9$ \\
$\beta_{2}$-agonist use inhalations·night ${ }^{-1}$ & $1.40 \pm 1.4$ & $1.25 \pm 0.8$ \\
Quality of life questionnaires & & \\
$\quad$ AQLQ & $5.66 \pm 0.80$ & $5.70 \pm 0.67$ \\
LWAQ & $0.61 \pm 0.21$ & $0.63 \pm 0.31$ \\
PGWB & $84.36 \pm 17.19$ & $85.31 \pm 14.99$ \\
SF36 & $72.89 \pm 17.27$ & $73.15 \pm 15.39$ \\
\hline
\end{tabular}

Values are shown as mean \pm SD. FEV1: forced expiratory volume in one second; PEF: peak expiratory flow; AQLQ: Asthma Quality of Life Questionnaire; LWAQ: Living with Asthma Questionnaire; PGWB: Psychological and General Well-Being scale; SF36: Short Form 36.

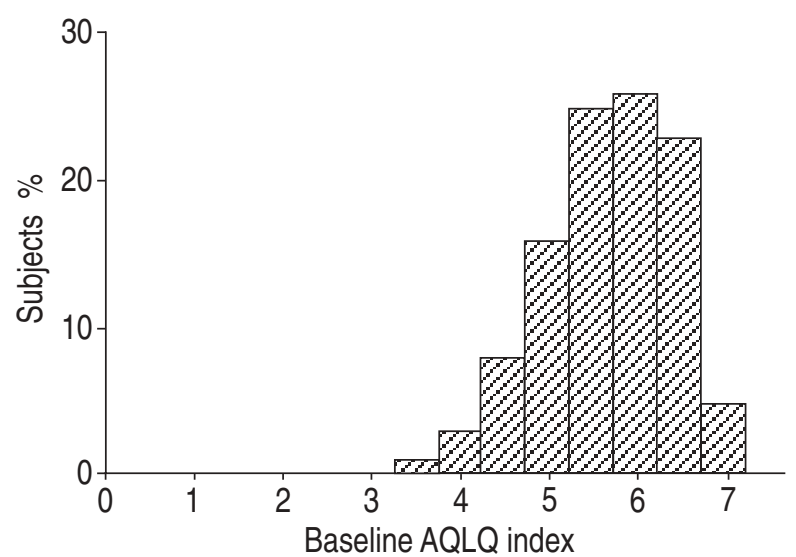

Fig. 1. - Frequency distribution of baseline values of the Asthma Quality of Life Questionnaire (AQLQ) $(n=110)$. AQLQ scale: 0-7, with 7 as the best score. Mean \pm SD: $5.7 \pm 0.74$.

witnarew auring tne treatment period (one adverse event and two noncompliance), along with four patients in the placebo group (all asthma deterioration).

\section{Clinical outcome measures and diary}

Treatment with formoterol improved all respiratory outcome measures. Mean FEV1 changed from 2.39 to $2.50 \mathrm{~L}$ $(\mathrm{p}>0.05)$ in the formoterol group, but was stable at 2.19 to $2.20 \mathrm{~L}$ in the placebo group. Mean use of inhaled terbutaline for the relief of symptoms decreased during treatment with formoterol $(-1.88$ inhalations, $\mathrm{p}<0.0001)$ and not during placebo (+0.26 inhalations). The difference in change in morning PEF between formoterol and placebo was 38.7 $\mathrm{L} \cdot \mathrm{min}^{-1}(\mathrm{p}<0.0001)$. Mean total daily symptoms in the formoterol group decreased from 3.8 to 3.0 but increased from 3.5 to 4.0 in the placebo group. The difference in change in daily mean total symptom scores between formoterol and placebo was $1.3(\mathrm{p}=0.012)$. Adverse events occurred at the same rate in both treatment groups.

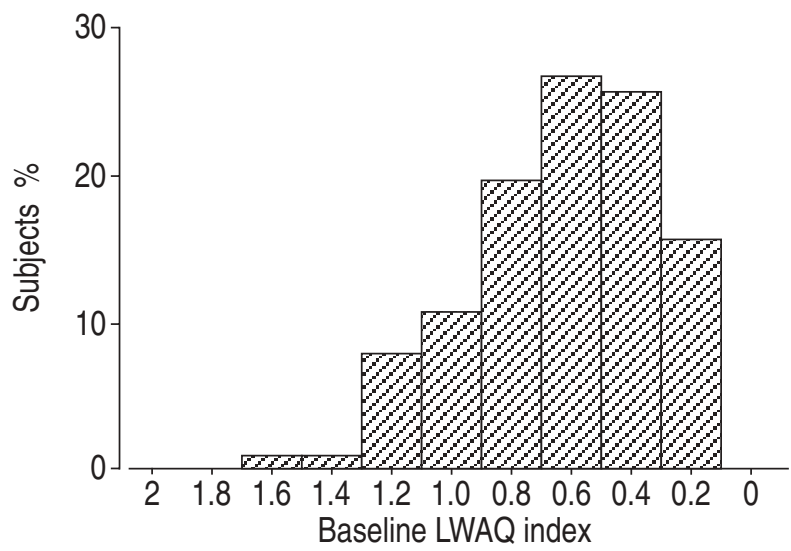

Fig. 2. - Frequency distribution of baseline values of the Living with Asthma Questionnaire (LWAQ) $(\mathrm{n}=110)$. LWAQ scale: $2-0$, with 0 as the best score. Mean \pm SD: $1.38 \pm 0.30$.

\section{Quality of life}

Baseline values of QoL are shown in table 1. There was no difference in QoL between the groups at the start of the study. The distribution of total baseline scores of the AQLQ and the LWAQ are shown in figures 1 and 2. The change in scores of the QoL questionnaires are shown in table 2. The LWAQ total score and the physical problem construct of the LWAQ improved significantly $(\mathrm{p}<0.05)$. The AQLQ domains and total score revealed the same trend in favour of the formoterol group, but the difference from the placebo group was not significant. Generic QoL measures did not show a significant improvement.

The effect sizes of the QoL questionnaires are shown in table 3. The effect size of the LWAQ total, the LWAQ physical and the symptom domain of the AQLQ were higher than the other QoL indices. Table 4 shows correlations between changes in QoL indices and changes in diary-obtained symptom scores, $\beta_{2}$-agonist use for the relief of symptoms, PEF and FEV1. The change in symptom scores obtained from the diary and the change in most of the QoL domains, with the exception of mental domains, were strongly related. Change in $\beta_{2}$-agonist use correlated more strongly with change in symptom-related domains of QoL than with change in mental-health dom-

Table 2. - Mean values for change of quality of life within patients during the treatment period for each treatment group

\begin{tabular}{lrrrrrrr}
\hline & \multicolumn{2}{c}{ Formoterol } & & \multicolumn{3}{c}{ Placebo } & \\
\cline { 2 - 3 } & Pre & Change & & Pre & Change & p-value* \\
\hline AQLQ & 5.66 & 0.15 & & 5.70 & 0.05 & 0.53 \\
Activity & 5.28 & 0.36 & & 5.40 & 0.25 & 0.65 \\
Symptoms & 5.50 & 0.19 & & 5.63 & -0.12 & 0.12 \\
Emotional & 6.26 & -0.02 & & 6.25 & 0.08 & 0.51 \\
Environment & 5.73 & 0.10 & & 5.70 & 0.14 & 0.80 \\
LWAQ & 0.61 & -0.05 & & 0.63 & 0.00 & 0.048 \\
Physical problem & 0.67 & -0.06 & & 0.71 & 0.01 & 0.044 \\
Emotional problem & 0.46 & -0.04 & & 0.44 & -0.01 & 0.35 \\
\hline SF36 total & 72.89 & 1.16 & & 73.15 & 1.20 & 0.99 \\
PGWB total & 84.36 & 0.49 & & 85.31 & 3.33 & 0.19 \\
\hline
\end{tabular}

*: Difference between the two treatment groups. AQLQ: Asthma Quality of Life Questionnaire; LWAQ: Living with Asthma Questionnaire; SF36: Short Form 36; PGWB: Psychological and General Well-Being scale. 
Table 3. - Effect sizes of changes in quality of life during treatment with formoterol in comparison to treatment with placebo

\begin{tabular}{lcc}
\hline & Effect size & $95 \%$ CI \\
\hline AQLQ & 0.128 & $-0.267-0.532$ \\
Activity & 0.106 & $-0.343-0.559$ \\
Symptoms & 0.316 & $-0.083-0.724$ \\
Emotional & 0.132 & $-0.266-0.534$ \\
Environment & 0.051 & $-0.347-0.451$ \\
LWAQ & 0.394 & $-0.003-0.796$ \\
Physical problem & 0.407 & $-0.015-0.809$ \\
Emotional problem & 0.196 & $-0.192-0.589$ \\
\hline SF36 total & 0.008 & $-0.380-0.397$ \\
PGWB total & 0.268 & $-0.120-0.664$ \\
\hline
\end{tabular}

CI: confidence interval; AQLQ: Asthma Quality of Life Questionnaire; LWAQ: Living with Asthma Questionnaire; SF36: Short Form 36; PGWB: Pscyhological and General Well-Being scale.

Table 4. - Correlations between changes in quality of life and changes in diary-obtained symptom scores, rescue $\beta_{2}$-agonist use, peak expiratory flow (PEF) and forced expiratory volume in one second $\left(F^{2} V_{1}\right)$ after 6 months of treatment

\begin{tabular}{lcccl}
\hline & Symptoms & $\beta$-agonist & PEF & FEV1 \\
\hline AQLQ & $-0.65^{+}$ & $-0.38^{+}$ & $0.49^{+}$ & $0.37^{+}$ \\
Activities & $-0.53^{+}$ & $-0.22^{* *}$ & $0.45^{+}$ & $0.26^{* *}$ \\
Symptoms & $-0.60^{+}$ & $-0.43^{+}$ & $0.52^{+}$ & $0.39^{+}$ \\
Emotions & $-0.51^{+}$ & $-0.24^{* *}$ & $0.25^{* *}$ & 0.09 \\
Environment & $-0.40^{+}$ & $-0.29^{* *}$ & $0.30^{* *}$ & $0.30^{* *}$ \\
LWAQ & $0.46^{+}$ & $0.32^{* *}$ & $-0.50^{+}$ & $-0.30^{* *}$ \\
Physical & $0.47^{+}$ & $0.28^{* *}$ & $0.54^{+}$ & $-0.32^{* *}$ \\
Mental & 0.17 & $0.25^{* *}$ & -0.19 & -0.13 \\
SF36 & $-0.44^{+}$ & -0.15 & $0.33^{+}$ & 0.14 \\
PGWB & $-0.38^{+}$ & -0.11 & 0.12 & 0.07
\end{tabular}

Values are shown as correlation coefficients. AQLQ: Asthma Quality of Life Questionnaire; LWAQ: Living with Asthma Questionnaire; SF36: Short Form 36; PGWB: Psychological and General Well-Being scale. ${ }^{* *}: \mathrm{p}<0.01,{ }^{+}: \mathrm{p}<0.0001$.

ains of QoL. The same applied to the correlations in lung function scores and QoL, with stronger correlations with change in symptom and physical domains than with change in mental-health domains. The change in AQLQ showed stronger relations with the change in symptoms, $\beta_{2}$-agonist use and FEV1 than the LWAQ. There was little change in the generic questionnaires.

\section{Discussion}

This study showed that all measurements of asthma, both subjective and objective, improved early in the study and maintained their improvement with a high statistical significance. However, only two QoL variables (the physical problem construct of the LWAQ and the LWAQ total score) showed an improvement which reached statistical significance. Calculation of effect sizes showed that LWAQ total, the physical construct of the LWAQ and the symptom domain of the AQLQ had almost equal responsiveness. The AQLQ total and the other domains of the AQLQ, as well as the generic QoL questionnaires, were unable to detect differences in QoL between the formoterol and placebo group. This was also reflected by low effect sizes, which also showed little responsiveness. Correlations between changes in asthma-specific QoL and changes in other subjective measures of asthma severity, such as symptom scores and need for $\beta_{2}$-agonist use, and objective measures, such as FEV1, were highly significant. The improvement in the physical problem domain of the LWAQ and the relatively strong correlation between clinical improvement and change in most domains of the AQLQ and the LWAQ shows that the asthma-specific QoL questionnaires showed a better longitudinal construct validity than the generic questionnaires.

RUtTen-van MöLKEn et al. [23] showed that improvement associated with salmeterol treatment was detected only by the three domains of AQLQ and not by the construct subscales of the LWAQ. In their opinion, the greater responsiveness of the AQLQ resulted from "informed administration", i.e. that patients read the answers they had given at baseline just before answering the questions at follow-up. This informed answering is recommended as a part of the procedure for this questionnaire [22]. In the present study this administration procedure was deliberately not followed since it was found to be in contrast to the administration procedures of the other questionnaires. This study therefore may add support to the hypothesis that "informed administration" improves the responsiveness of the AQLQ. Whether other questionnaires could also improve their responsiveness by using "informed administration" remains unclear.

In a study in which the use of salmeterol and low-dose inhaled corticosteroids were compared with high-dose inhaled corticosteroids in asthma patients, problem scores obtained from structured QoL diaries proved to be more sensitive to change than QoL quesionnaires [24]. In the present study a symptom-score diary was used, with very short simple questions about cough, wheeze, activity, chest discomfort and sputum production. These diaries were filled in during the whole study and it must be considered that patients always saw their recent previous answers, thus obtaining partial "informed administration". This indicates that a simple diary, as used in our study with informed administration, provides a more sensitive instrument to detect changes in subjective symptoms than asthma-specific QoL questionnaires. However, QoL questionnaires provide information other than diary-obtained symptom scores, $\beta_{2}$-agonist use and FEV1. QoL, defined as patients' functioning and subjective wellbeing, is a complex and abstract construct that is influenced by many aspects of life, including asthma. In the present group of patients with near-normal health-related QoL, the impact of the change in lung function on the symptom scores and the use of $\beta_{2}$-agonists did not have a comparable influence on QoL. The influence of these changes may disappear in the noise of other factors that were perceived as being more important. This study suggests that an improvement of $>\sim 4 \%$ in $\mathrm{FEV}_{1}$ or 1.3 points in symptom scores is needed in order to gain substantial improvement in QoL.

Furthermore, many chronic patients adapt over time to changes in the disabilities of their chronic disease. In this study QoL was assessed with an interval of 6 months, a period which could have been long enough for the patients to adapt to their new situation.

Improvements in QoL during treatment with formoterol were limited in these patients, despite an improvement of diary-obtained symptom scores and lung function. LWAQ total score, the LWAQ physical construct score and the AQLQ symptom domain were responsive to change. Chan- 
ges in symptom scores, PEF and FEV1 correlated significantly with changes in LWAQ and AQLQ, showing a good longitudinal construct validity. Generic QoL measurements of the SF36 and the PGWB showed no improvement and correlated less with changes in symptoms and measurements of pulmonary function.

Therefore, from this study on the effect of formoterol in addition to inhaled corticosteroids in patients with mild asthma, it can be concluded that the responsiveness to change of the total score on the Living with Asthma Questionnaire was better than that on the Asthma Quality of Life Questionnaire and the generic quality of life questionnaires. The longitudinal construct validity of the Asthma Quality of Life Questionnaire was similar to the Living with Asthma Questionnaire and superior to the Short Form 36 and the Psychological and General Well-Being scale.

Acknowledgements: The authors would like to thank the Dutch formoterol investigators, A.J.M. Schreurs (Amsterdam), H.H. Berendsen (Den Haag), J.L.M. van Helmond (Roosendaal) and P.M. Hooghiemstra (Amsterdam), as well as the Dutch general practitioners who co-operated in this study.

\section{References}

1. Guyatt GH, Veldhuyzen Van Zanten SJO, Feeny DH, Patric DL. Measuring quality of life in clinical trials: a taxonomy and review. Can Med Assoc J 1989; 140: 1441-1448.

2. Hyland ME, Kenyon CAP, Jacobs PA. Sensitivity of quality of life domains and constructs to longitudinal change in a clinical trial comparing salmeterol with placebo in asthmatics. Qual Life Res 1994; 3: 121-126.

3. Noonan M, Chervinsky P, Busse WW, et al. Fluticasone propionate reduces oral prednisolone use while it improves asthma control and quality of life. Am J Respir Crit Care Med 1995; 152: 1467-1473.

4. Maill AR, Kaptein AA, de Haes JCJM, Everaerd WThAM. Assessing quality of life in chronic non-specific lung disease: a review of empirical studies published between 1980 and 1994. Qual Life Res 1996; 5: 287-301.

5. Corsico R, Patruno V, Fracchia C. Anti-asthma drugs and quality of life. Eur Respir Rev 1993; 14: 366-368.

6. Lahdensuo A, Haahtela T, Herrala J, et al. Randomised comparison of guided self-management and traditional treatment of asthma over one year. Br Med J 1996; 312: 748-752.

7. Greening AP, Ind PW, Northfield M, Shaw G, Allen \& Hanburys Ltd UK Study Group. Added salmeterol versus higher-dose corticosteroid in asthma patients with symptoms on existing inhaled corticosteroid. Lancet 1994; 344: 219-224.

8. Arvidsson P, Larsson S, Löfdahl CG, Melander B, Svedmyr N, Wåhlander L. Inhaled formoterol during one year in asthma: a comparison with salbutamol. Eur Respir $J$ 1991; 4: 1168-1173.
9. Kesten S, Chapman KR, Broder I, et al. Sustained improvement in asthma with long term use of formoterol fumerate. Ann Allergy 1992; 69: 415-420.

10. Kesten S, Chapman KR, Broder I, et al. A three-month comparison of twice daily inhaled formoterol versus four times daily inhaled albuterol in the management of stable asthma. Am Rev Respir Dis 1991; 144: 622-625.

11. Pearlman DS, Chervinsky P, LaForce C, et al. A comparison of salmeterol with albuterol in the treatment of mild-to-moderate asthma. N Engl J Med 1992; 327: 1420 1425 .

12. Rowe BH, Oxman AD. Performance of an asthma quality of life questionnaire in an outpatient setting. Am Rev Respir Dis 1993; 148: 675-681.

13. van der Molen T, Postma DS, Turner MO, et al. Effects of the long-acting $\beta$-agonist formoterol on asthma control in asthmatic patients using inhaled corticosteroids. Thorax 1997; 52: 535-539.

14. van der Molen T, Postma DS, Schreurs AJM, Bosveld HEP, Sears MR, Meyboom de Jong B. Discriminative aspects of two generic and two asthma specific instruments: relation with symptoms, bronchodilator use and lung function in patients with mild asthma. Qual Life Res 1997; 6: 353-361.

15. Ware JE, Sherbourne CD. The MOS-36-item Short-form Health Status Survey(SF-36): 1. Conceptual framework and item selection. Med Care 1992; 30: 473-483.

16. Ware JE, Kosinski M, Bayliss MS, McHorny CA, Rogers WH, Raczec A. Comparison of methods for the scoring and statistical analysis of SF36 health profile and summary measures: summary of results from the medical outcomes study. Med Care 1995; 33: 264-279.

17. Kamman R, Flett R. A scale to measure current level of general happiness. Aust J Psychol 1983; 35: 259-265.

18. Wan TTH, Liviratos B. Interpreting a general index of subjective well-being. Milbank Mem Fund 1978; 56: 531556.

19. Hyland ME, Finnis S, Irvine SH. A scale for assessing quality of life in adult asthma sufferers. $J$ Psychosom Res 1991; 35: 99-110.

20. Hyland ME. The living with asthma questionnaire. Respir Med 1991; 85: 13-16.

21. Kaptein AA, Brand PLP, Dekker FW, et al. Qualityof-live in a long-term multicentre trial in chronic nonspeciffc lung disease: assessment at baseline. Eur Respir $J$ 1993; 6: 1479-1484.

22. Juniper EF, Guyatt GH, Epstein RS, Ferrie PJ, Jaeschke R, Hiller TK. Evaluation of impairment of health related quality of life in asthma: development of a questionnaire for use in clinical trials. Thorax 1992; 47: 76-83.

23. Rutten-van Mölken MPMH, Custers F, Van Doorslaar EKA, et al. Comparison of performance of four instruments in evaluating the effects of salmeterol on asthma quality of life. Eur J Respir Dis 1995; 8: 888-898.

24. Hyland ME, Crocker GR. Validation of an asthma quality of life diary in a clinical trial. Thorax 1995; 50: 724-730. 